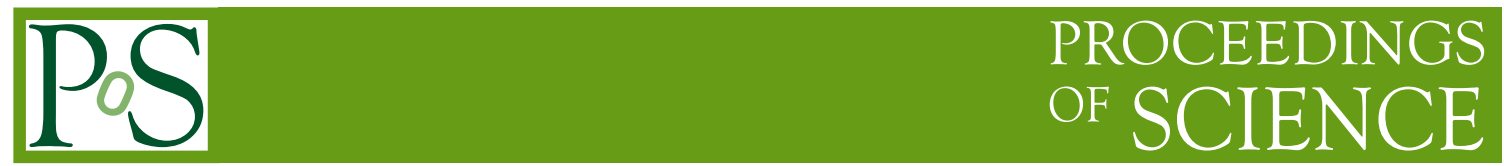

\title{
$\mathbf{B} \rightarrow \mu \mu$ and $\mathbf{B} \rightarrow \tau \nu$ Decays
}

\section{Fabrizio Scuri*}

I.N.F.N. - Sezione di Pisa - Italy

E-mail: fabrizio.scuriepi.infn.it

on behalf of the BaBar, Belle, CDF and Do Collaborations

An overview of the most recent experimental results on Branching Fractions of rare fully leptonic B decays is given; constraints on the parameters of some New Physics models are presented. Perspectives with new accelerator programs are discussed.

Flavor Physics and CP Violation 2009

May 27-June 12009

Lake Placid, NY, USA

* Speaker. 


\section{Introduction}

The sensitivity of recent experimental studies on rare purely leptonic decays of the B (and D) meson(s) performed at the B factories and at the Tevatron approaches the Standard Model predictions and opens a great opportunity to probe New Physics in various supersymmetric scenarios and, more generally, in any extension of the Standard Model which requires at least one additional Higgs doublet.

Treatment is focused on the $\mathrm{B} \rightarrow \tau \nu$ and on the $\mathrm{B}^{0} \rightarrow \mu \mu$ decay modes. For sake of completeness, one has to mention here that the Flavor Changing Neutral Current sector has been studied also in the $\mathrm{D}^{0} \rightarrow \mu \mu$ decay mode by the Hera-B, BaBar, and CDF collaborations. Very suppressed in the Standard Model due to the GIM mechanism (no top loop, decay rate dominated by long distance contributions, $B F\left(D^{0} \rightarrow l^{+} l^{-}\right) \simeq 10^{-13}$, this mode is sensitive to enhancements possible in R-Parity violating SUSY models [1]. CDF gave the most recent and accurate result on $B F\left(D^{0} \rightarrow \mu \mu\right)$, which translate into the following limit on the R-Parity violation couplings: $\lambda_{21 k} \lambda_{22 k}<9.8 \times 10^{-4}[2]$.

An overview of the experimental standing for the measurements of $B F\left(B^{-} \rightarrow \tau^{-} \bar{v}\right)$ and of $B F\left(B^{0} \rightarrow \mu^{+} \mu^{-}\right)$made at the $\mathrm{B}$ factories and at the Tevatron, respectively, is given in the following. Constraints from the present results on the parameters of some New Physics models are also given and the perspectives with the incoming new accelerator programs are discussed.

\section{Charged current in $\mathbf{B} \rightarrow \tau v$ decays}

The purely leptonic decay $B^{-} \rightarrow \tau^{-} \bar{v}$ proceeds in the Standard Model at tree level through quark annihilation into a $\mathrm{W}$ boson and it is of particular interest since it provides a direct measurement of the product of the CKM matrix element $V_{u b}$ and the B meson decay constant $f_{B}$. The Standard Model expression of the branching fraction is given by

$$
B F\left(B^{-} \rightarrow \tau^{-} \bar{v}\right)_{S M}=\frac{G_{F}^{2} m_{B} m_{\tau}^{2}}{8 \pi}\left(1-\frac{m_{\tau}^{2}}{m_{B}^{2}}\right) f_{B}^{2}\left|V_{u b}\right|^{2} \tau_{B},
$$

where $G_{F}$ is the Fermi coupling constant, $m_{B}$ and $m_{\tau}$ are the B meson and $\tau$ lepton masses, and $\tau_{B}$ is the $B^{-}$meson lifetime. Helicity conservation suppresses the electron and muon channels in the dependence on the lepton mass.

Determinations of the SM expected branching fraction from other experimental constraints are available from the UT fit and CKM fitter Collaborations; the expected SM values are the following:

$$
\begin{gathered}
B F^{U T f i t}\left(B^{-} \rightarrow \tau^{-} \bar{v}\right)=(0.83 \pm 0.12) \times 10^{-4} \\
B F^{C K M f i t t e r}\left(B^{-} \rightarrow \tau^{-} \bar{v}\right)=\left(0.80_{-0.09}^{+0.15}\right) \times 10^{-4}
\end{gathered}
$$

Details on the methods used to determine the values in equations 2.2 and 2.3 can be found by visiting the UT fit and $C K M$ fitter Collaboration websites ${ }^{1}$.

Recent experimental results on $B F\left(B^{-} \rightarrow \tau^{-} \bar{v}\right)$ are provided by the BaBar and Belle Collaborations from the analysis of large amounts of $\mathrm{B}^{+} \mathrm{B}^{-}$pair decays produced in $\mathrm{e}^{+} \mathrm{e}^{-}$collisions at

\footnotetext{
${ }^{1}$ http : //www.ut fit.org - http: //www.ckmfitter.in $2 p 3 . f r$
} 
the B factories. The analysis concepts are the following: one B meson of the initial pair is used in the tagging side and reconstructed through the hadronic or semi-leptonic decay modes; in the signal side, the $\tau$ daughter particle(s) is(are) detected; at least two neutrinos appear in the $B \rightarrow \tau \nu$ cascade. An additional requirement is that no particle is left after removing the decay products of tagging $\mathrm{B}$ and of the particle(s) from the $B \rightarrow \tau \nu$ decay. The most discriminating observable to reject background is the so called extra energy $\left(\mathrm{E}_{E C L}\right)$, i.e. the sum of energy clusters not associated to particles from tagging and signal $\mathrm{B}$ mesons.

The extra energy spectra from Belle and BaBar analyses are shown in Figure 1; an excess with respect to the background expectation is seen in the low energy part of the histograms.
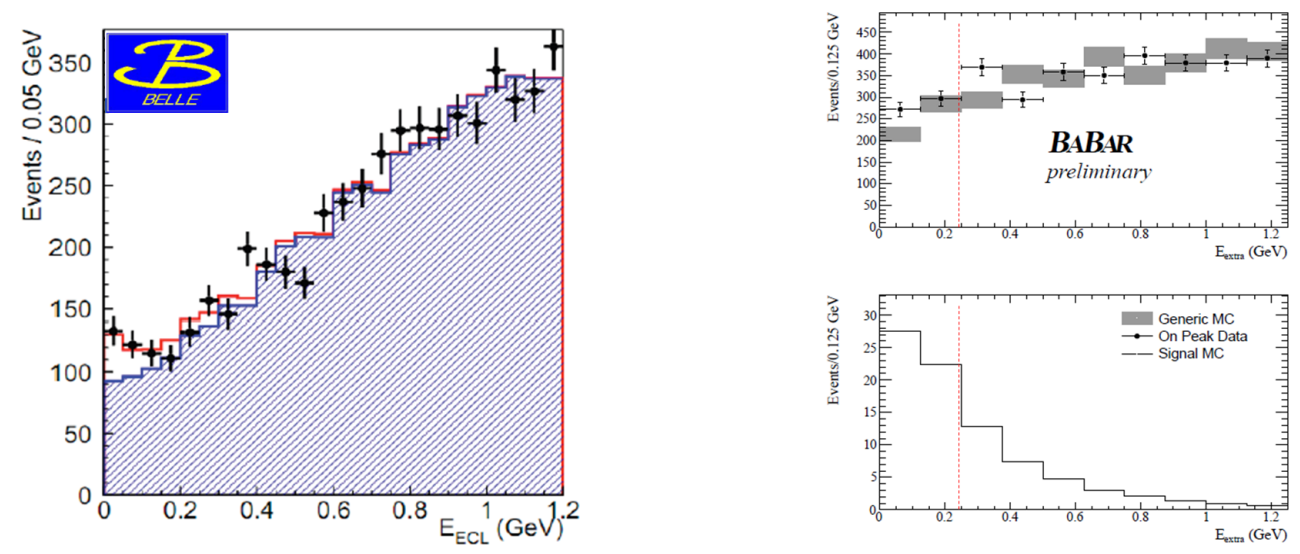

Figure 1: Extra energy spectra; Belle (left): points with error bar are data, solid hatched histogram is background, open red histogram is signal. BaBar (right): the signal region is on the left of the vertical dashed red line.

Most recent results on $B F\left(B^{-} \rightarrow \tau^{-} \bar{v}\right)$, including all $\tau$ decay modes, and published by BaBar and Belle, were obtained by using semi-leptonic decays of the tagging B meson. Tagging B candidates were selected by discriminating on the lepton momentum and on the opening angle between the tagging $\mathrm{B}$ and the $\mathrm{D}^{0(*)}$-lepton system. Belle found 154 signal events in a $657 \times 10^{6}$ BB pair sample [3] and the measured value was $B F\left(B^{-} \rightarrow \tau^{-} \bar{v}\right)=\left(1.65_{-0.37}^{+0.38}(\text { stat } .)_{-0.37}^{+0.35}(\right.$ syst. $) \times 10^{-4}$. BaBar analyzed some $459 \times 10^{6} \mathrm{BB}$ pairs observing 610 events and estimating $521 \pm 31$ background events [4]; the measured value was $B F\left(B^{+} \rightarrow \tau^{+} v\right)=(1.8 \pm 0.8$ (stat. $) \pm 0.1($ syst. $) \times 10^{-4}$. A summary of the BaBar and Belle experimental results for semil-eptonic and hadronic decays of the tagging B is shown in Table 1.

\begin{tabular}{|c|c|c|}
\hline \multicolumn{3}{|c|}{$B F\left(B^{-} \rightarrow \tau^{-} \bar{v}\right) \times 10^{4}$} \\
\hline & hadronic tag & semi-leptonic tag \\
\hline BaBar & $1.8_{-0.8}^{+0.9}($ stat $) \pm 0.4($ bkg $) \pm 0.2($ syst $)[5]$ & $1.8 \pm 0.8($ stat $) \pm 0.1($ syst $)$ \\
Belle & $1.79_{-0.49}^{+0.56}(\text { stat })_{-0.51}^{+0.46}($ syst $)[6]$ & $1.65_{-0.37}^{+0.38}(\text { stat })_{-0.37}^{+0.35}($ syst $)$ \\
\hline
\end{tabular}

Table 1: B factories result summary

Most recent results from B factories (semi-leptonic tag) produced a sizable increase in the 
experimental average value of $B F\left(B^{-} \rightarrow \tau^{-} \bar{v}\right)$ with respect to the previous world averages as summarized in Table 2.

\begin{tabular}{|c|c|}
\hline \multicolumn{2}{|c|}{$B F\left(B^{-} \rightarrow \tau^{-} \bar{v}\right) \times 10^{4}$} \\
\hline PDG '08 & $1.4 \pm 0.4$ \\
HFAG '08 & $1.51 \pm 0.33$ \\
\hline New BaBar average & $1.80 \pm 0.65$ \\
New Belle average & $1.70 \pm 0.42$ \\
\hline New world average & $1.74 \pm 0.34$ \\
\hline
\end{tabular}

Table 2: World averages for $B F\left(B^{-} \rightarrow \tau^{-} \bar{v}\right)$

Because of the new world average for $\sin 2 \beta$, which is smaller than before [7], and of the new world average for $B F\left(B^{-} \rightarrow \tau^{-} \bar{v}\right)$, a new tension in the CKM global fit has appeared. This can be seen on the $(\sin 2 \beta, \mathrm{BR}(\mathrm{B} \rightarrow \tau \nu)$ plane (Figure $2 \mathrm{a})$ ) regarding the prediction from the global fit without using these measurements. The tension can be quantified as a 2.4 sigma deviation by comparing measurement and indirect fit prediction for $\mathrm{BR}(\mathrm{B} \rightarrow \tau v)($ figure $2 \mathrm{~b})$ ).
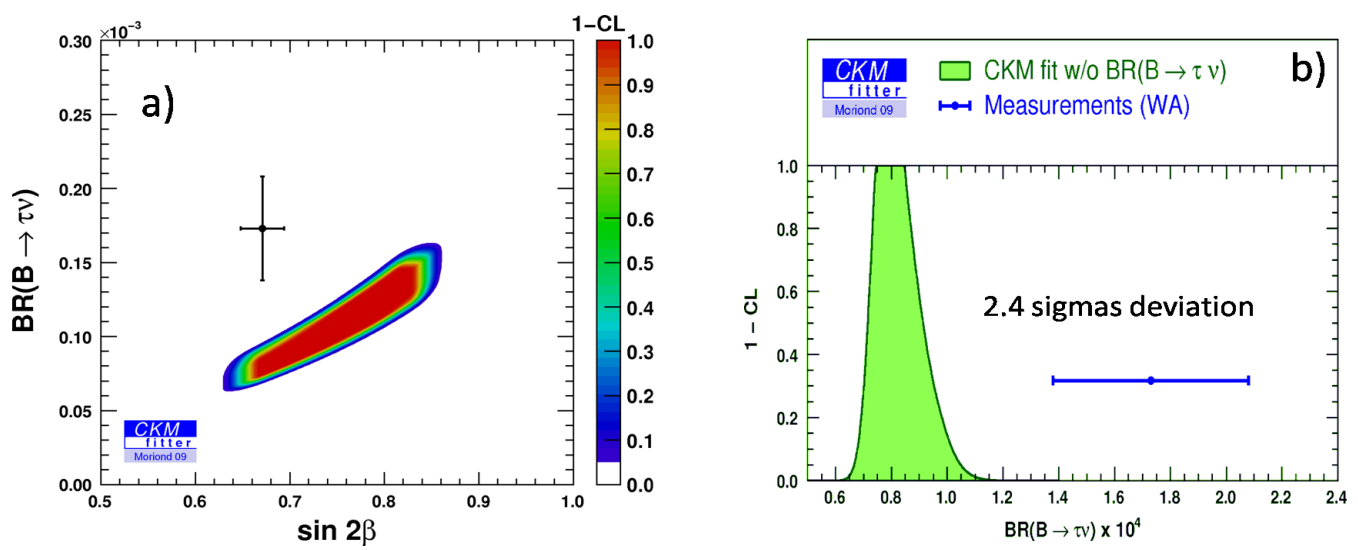

Figure 2: a) prediction from the global fit without using $\mathrm{BF}(B \rightarrow \tau v)$ and $\sin 2 \beta$ measurements; the cross corresponds to the experimental values with $1 \sigma$ experimental uncertainties; $b$ ) indirect fit prediction and measured value for $\mathrm{BF}(B \rightarrow \tau v)$

Purely leptonic B decays are of particular interest also to test New Physics effects; new flavor changing interactions are generated already at tree level via the exchange of a charged Higgs boson by supersymmetric extensions of the Standard Model or, more generally, by extensions which require at least one additional Higgs doublet. Charged Higgs can enhance or suppress the branching fraction [8] by modifying the Standard Model expectation with the $\mathrm{m}_{l}$ independent factor $\mathrm{r}_{H}$ defined below:

$$
B F(B \rightarrow \tau v)=B F(B \rightarrow \tau \nu)_{S M} \times\left(1-r_{H}^{2}\right)^{2} ; \quad r_{H}=\frac{m_{B}}{m_{H}} \times \tan \beta
$$

The $B F(B \rightarrow \tau v)$ value is enhanced with respect to the Standard Model prediction if $\tan \beta>0.27$ $\mathrm{m}_{H}(\mathrm{GeV})$. 
In the minimal supersymmetric extension of the standard model (MSSM), the effective scalar coupling $g_{s}$ has an additional dependence on sparticle mass parameters for large values of $\tan \beta$ [9], expressed by the sparticle loop factors $\varepsilon_{0, \tau}$ in the following equations:

$$
\begin{array}{r}
B F(B \rightarrow \tau v)=B F(B \rightarrow \tau v)_{S M} \times\left(1-g_{s}\right)^{2} \\
g_{s}=\frac{m_{B}^{2}}{m_{H}^{2}} \times \frac{\tan ^{2} \beta}{\left(1+\varepsilon_{0} \tan \beta\right)\left(1+\varepsilon_{\tau} \tan \beta\right)}
\end{array}
$$

The limits on New Physics contributions to $B F(B \rightarrow \tau v)$ set by the new world experimental average are shown in Figure 3 for the 2HDM (two Higgs doublet model) and the MSSM.
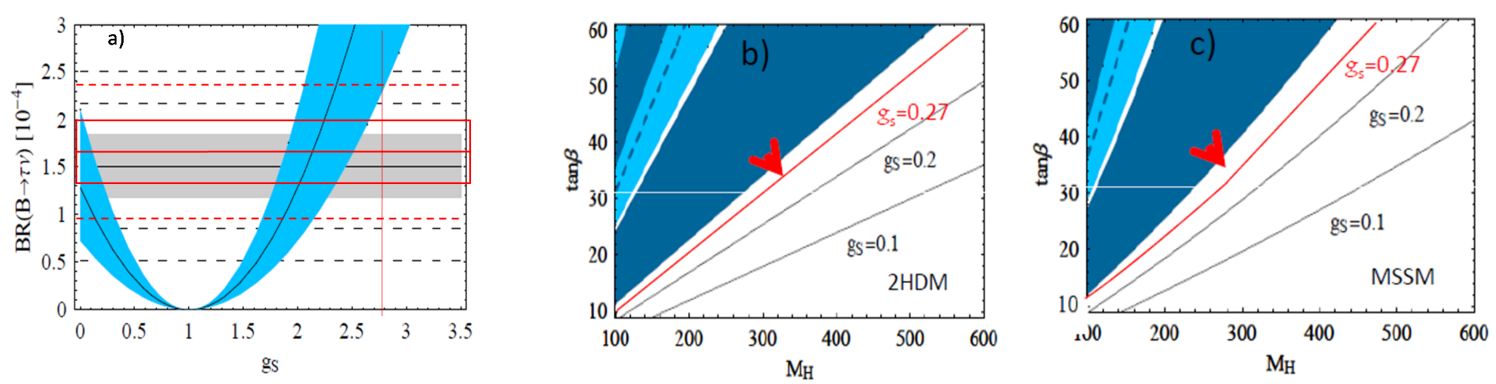

Figure 3: a) predicted $B F(B \rightarrow \tau \nu)$ (blue band) as a function of $g_{s}$ (defined in text); the gray band defines the $1 \sigma$ region around the HFAG' 08 w.a., the red box defines the $1 \sigma$ region around the new BaBar and Belle average (Moriond '09); b) and c) exclusion zones in the $\left(M_{H}, \tan \beta\right)$ plane from $\mathrm{BF}(B \rightarrow \tau v)$ in a $2 \mathrm{HDM}$ (b) and in the MSSM (c) with $\varepsilon_{0}=0.01$ and $\varepsilon_{\tau} \simeq 0$. The blue region is the $95 \%$ C.L. exclusion region from the HFAG'08 BF w.a., the red line corresponds to the $90 \%$ C.L. border of the exclusion region from the new average.

\section{Flavor changing neutral current in muonic $\mathbf{B}^{0}$ and $\mathbf{D}^{0}$ decays}

Flavor changing neutral current (FCNC) decays are very suppressed within Standard Model; they are forbidden at tree level and they can proceed only through loop diagrams; quite accurate predictions of $B F\left(B_{s, d}^{0} \rightarrow \mu^{-} \mu^{-}\right)$within Standard Model have been presented in a recent review [10]:

$$
\begin{gathered}
B F\left(B_{s}^{0} \rightarrow \mu^{-} \mu^{-}\right)=(3.6 \pm 0.3)^{-9} \\
B F\left(B_{d}^{0} \rightarrow \mu^{-} \mu^{-}\right)=(1.1 \pm 0.1)^{-10}
\end{gathered}
$$

Present experimental sensitivity to $B F\left(B^{0} \rightarrow \mu^{-} \mu^{-}\right)$is about one order of magnitude above the Standard Model predicted value; however, FCNC decays are very sensitive to New Physics which can appear:

- at tree level through R-Parity violation in SUSY (Figure 4);

- through loops in:

a) Minimal Flavor Violation (MFV) extensions of the Standard Model such as 2HDM; 

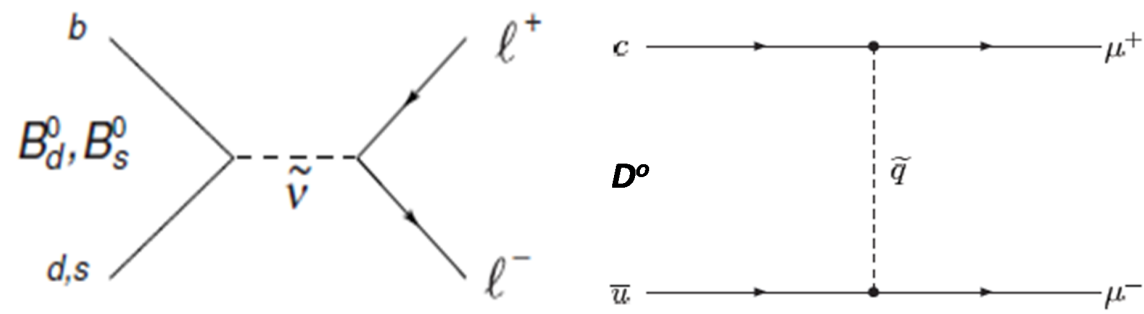

Figure 4: Examples of Feynman diagrams of R-Parity violation for leptonic decays of $\mathrm{B}^{0}$ and $\mathrm{D}^{0}$ mesons.
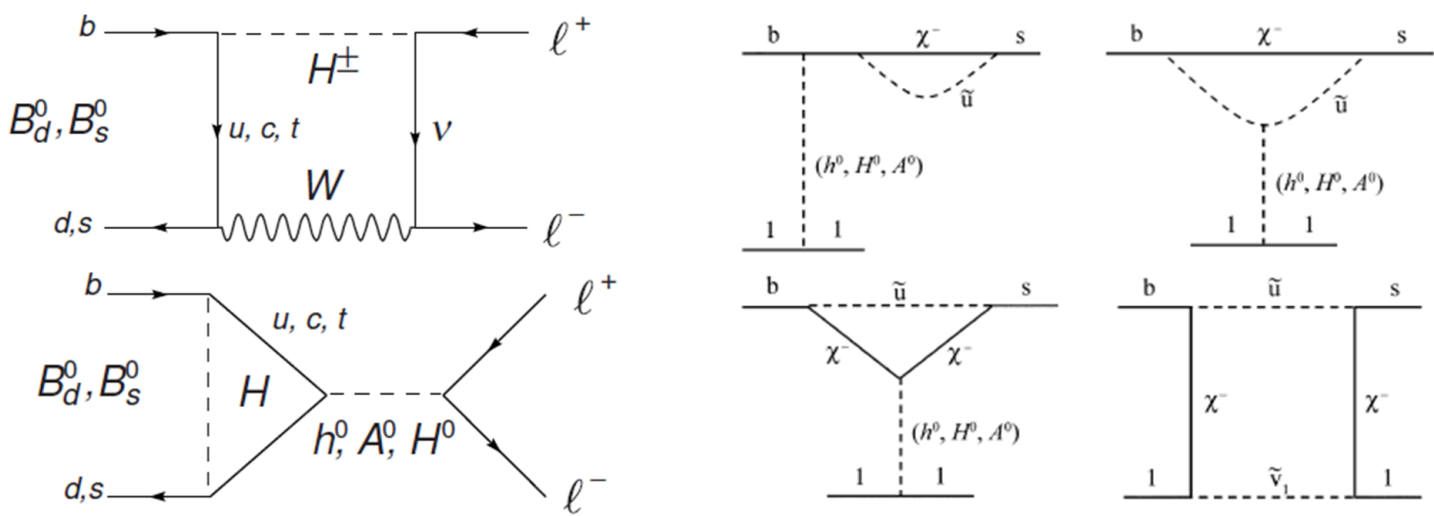

Figure 5: Examples of Feynman loop diagrams of FCNC contributions from New Physics, 2HDM (left) and MSSM (right).

b) MSSM where the contribution from New Physics to the branching fraction is expected to grow with $\tan \beta$ as $B F^{S U S Y}(B \rightarrow \mu \mu) \propto(\tan \beta)^{6}$ (Figure 5)

Therefore, it's of particular interest to follow the experimental program at the Tevatron where the sensitivity on $B F$ of the combined results of the CDF and D0 experiments is expected to be $10^{-8}$.

Experimental procedures are similar for the two Tevatron experiments; the general strategy common to CDF and D0 is the following: a blind optimization of the event selection in the dimuon trigger data samples is performed by using simulated data in the signal region of the dimuon invariant mass and real data in the signal sidebands; the $B^{+} \rightarrow J / \Psi K^{+}$decay is used as the normalization mode; signal and normalization mode are reconstructed in the same data sample and use the same criteria to minimize systematic effects. The background in the signal region is estimated by extrapolation from the sidebands, after cut optimization; the final step is the event count in the "unblind" signal region. Different approaches to the statistical treatment are used by the two experiments to set upper limits on the $B F$ when comparing the observed and the expected event numbers in the signal region; details of the methods can be found in references [11] and [12].

The dimuon invariant mass distributions of $\mathrm{D} 0$ and $\mathrm{CDF}$ analyses with $2 \mathrm{fb}-1$ are shown in Figure 6 . The D0 analysis is divided in two data taking periods, run-IIa and run-IIb, corresponding 
to different set-ups of the inner tracking detector. Different cuts on the event likelihood ratio are applied in the two periods, where $1(2)$ event(s) was(were) observed with $0.8 \pm 0.2(1.5 \pm 0.5)$ expected events for run-IIa(run-IIb). CDF plots are relative to the case where both muons are detected in the Central MUon detector (CMU-CMU) and to the case where one muon is central and one is detected in the Central Muon eXtension (CMU-CMX). CDF observed 3 events with $3.5 \pm 0.3$ expected events in the $\mathrm{B}_{s}$ signal region and observed 6 events with $4.0 \pm 0.3$ expected events in the $\mathrm{B}_{d}$ signal region.
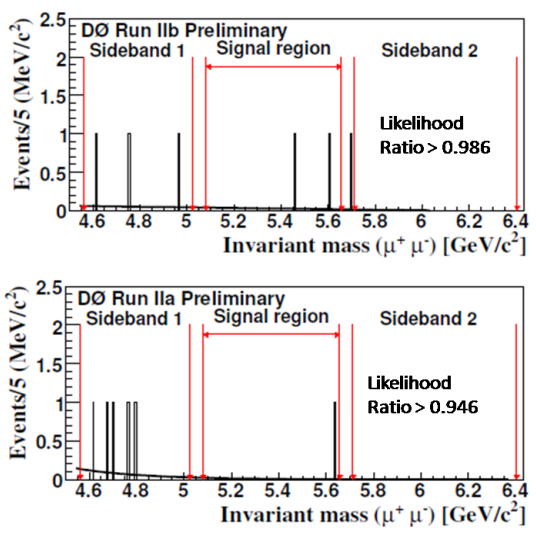
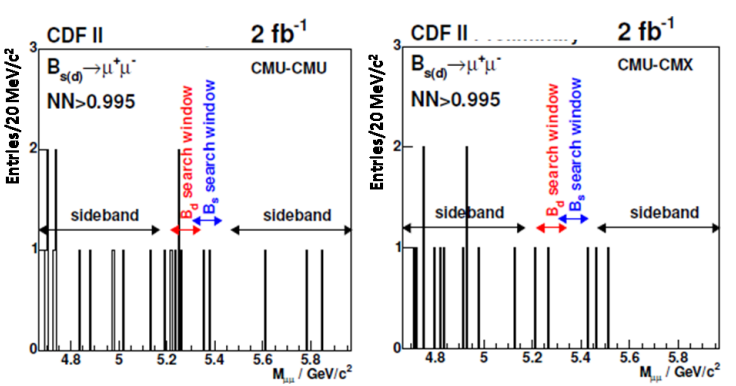

Figure 6: Dimuon invariant mass spectra; left: D0 results for run-IIa and run-IIb; right: CDF results for the two central muons (CMU-CMU) case and for the CMU-CMX (one muon in the Central Muon eXtension) case.

The most recent published results on $B F\left(B_{d, s}^{0} \rightarrow \mu^{-} \mu^{-}\right)$are summarized in Table 3, including $\mathrm{B}$ factories and Tevatron experiments.

\begin{tabular}{|c|c|c|}
\hline \multicolumn{3}{|c|}{$90 \%(95 \%)$ C.L. limits $\times 10^{8}$} \\
\hline & $\mathrm{B}_{s}^{0}$ & $\mathrm{~B}_{d}^{0}$ \\
\hline BaBar (ref. [13]) & $\mathrm{n} / \mathrm{a}$ & 5.2 \\
$\mathrm{D} 0\left(2 \mathrm{fb}^{-1}\right)$ & $7.5(9.3)$ & $\mathrm{n} / \mathrm{a}$ \\
$\mathrm{CDF}\left(2 \mathrm{fb}^{-1}\right)$ & $4.7(5.8)$ & $1.5(1.8)$ \\
\hline
\end{tabular}

Table 3: Limits on $B F\left(B^{0} \rightarrow \mu^{+} \mu^{-}\right)$

The trend of the experimental sensitivity on $B F\left(B_{s}^{0} \rightarrow \mu^{+} \mu^{-}\right)$as a function of the integrated luminosity at the Tevatron is shown in Figure $7 \mathrm{a}$ ). The red triangle corresponds to the announced D0 expected limit from a new analysis with $4.8 \mathrm{fb}^{-1}$ integrated luminosity [14]; extrapolation to the $10 \mathrm{fb}^{-1}$ value, to be reached at the end of the Tevatron operation, leads to a combined sensitivity close to $10^{-8}$.

Among various supersymmetric scenarios which can be tested, the rare $B F\left(B_{s}^{0} \rightarrow \mu^{+} \mu^{-}\right)$ decay mode can probe the parameter space of the minimal super gravity model (mSUGRA); in Figure $7 \mathrm{~b})$ is shown the allowed region in the plane $\left(m_{0}-m_{1 / 2}\right)$, the universal scalar and gaugino masses at the grand unification scale $\left(M_{G}=10^{16} \mathrm{GeV}\right)$. Note that combined measures of $B F\left(B_{s}^{0} \rightarrow\right.$ 
$\mu^{+} \mu^{-}$) from Tevatron at $10 \mathrm{fb}^{-1}$ and of the deviation from Standard Model of the muon anomalous magnetic momentum $\left(a_{\mu}\right)$ could completely rule-out mSUGRA.
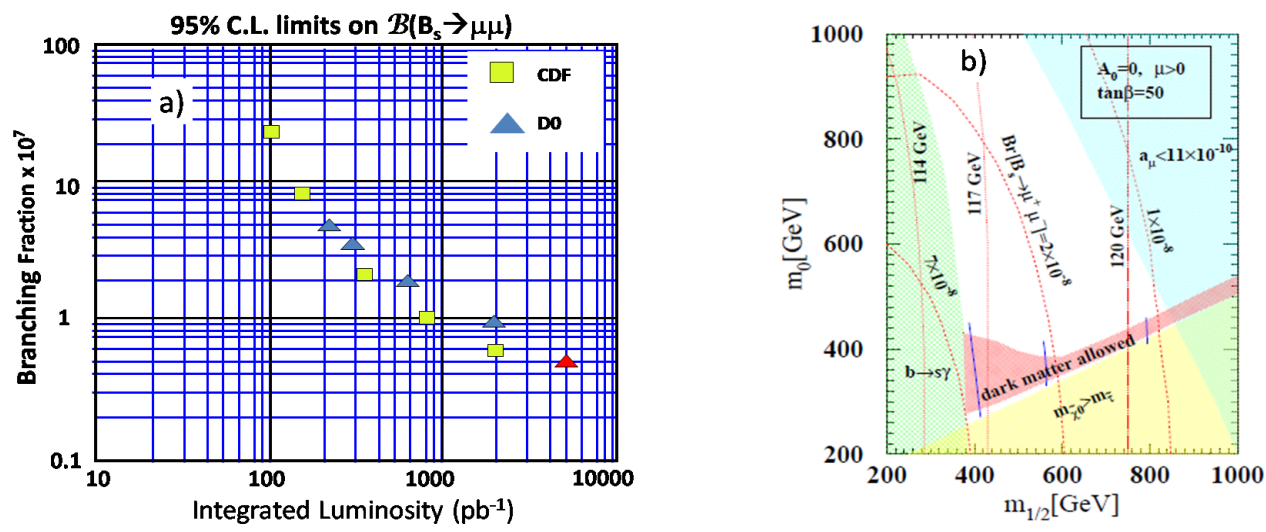

Figure 7: a) $95 \%$ C.L. limits on $B F\left(B_{s} \rightarrow \mu \mu\right)$ from Tevatron experiments; the red triangle corresponds to the D0 expected limit with $4.8 \mathrm{fb}^{-1}$; b) $B F\left(B_{s} \rightarrow \mu \mu\right)$ in the $m_{0}-m_{1 / 2}$ plane for $\tan \beta=50$; other mSUGRA parameters are fixed to be $A_{0}=0$ and $\mu>0$. Plot is taken from ref. [15].

\section{Areas of improvement and perspectives with the new accelerator programs}

The experimental sensitivity on the measurements of the $B^{-} \rightarrow \tau^{-} \bar{v}$ decay at the B factories is limited by the low statistics, due to the unavoidably low efficiency $\left(\mathrm{O}\left(10^{-3}\right)\right.$. The systematic error due to the background estimation is also large. A sizable gain in accumulated statistics will be obtained with the designed Super B factories where the integrated luminosity at the end of operations will be a factor of 25 greater than that of the present $B$ factories. The expected relative error on $B F\left(B^{-} \rightarrow \tau^{-} \bar{v}\right)$ is $3 \%-4 \%$ at $75 \mathrm{ab}^{-1}$ [16].

The $B_{s, d}^{0} \rightarrow \mu^{+} \mu^{-}$decay modes are among the golden channels for New Physics discovery at the Tevatron; the expected limit of the CDF and D0 combined results on $B F\left(B_{s}^{0} \rightarrow \mu^{+} \mu^{-}\right)$ at $10 \mathrm{fb}^{-1}$ (end of the Tevatron Run-II) is close to $1 \times 10^{-8}$, just a factor 3 above the Standard Model expectation. This goal should be achieved by exploiting the full available statistics and by improving the particle identification and the acceptance.

A limit somewhat better than the Tevatron should be achieved at the Super B factories; estimated sensitivities are the following [16], [17]:

$$
\begin{aligned}
& B F\left(B_{s}^{0} \rightarrow \mu^{-} \mu^{-}\right)(\Upsilon(5 S))<8 \times 10^{-9} \text { at } 30 \mathrm{ab}^{-1} \\
& B F\left(B_{d}^{0} \rightarrow \mu^{-} \mu^{-}\right)(\Upsilon(4 S))<7 \times 10^{-9} \text { at } 50 \mathrm{ab}^{-1}
\end{aligned}
$$

Sensitivity close to the Standard Model branching fraction value should be reached in the first years of operation of the LHC collider; the estimated sensitivity of the LHCb experiment [18] as a function of the integrated luminosity is shown in Figure 8. 


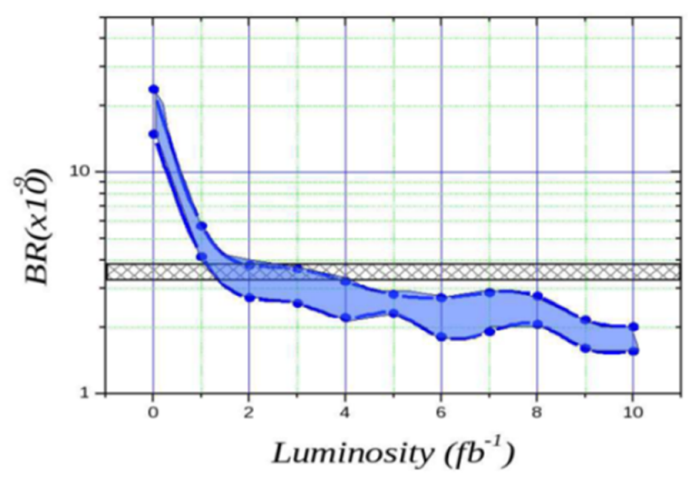

Figure 8: $\mathrm{LHCb} 3 \sigma$ sensitivity on $B F\left(B_{s}^{0} \rightarrow \mu^{+} \mu^{-}\right)$measurement as a function of the integrated luminosity; the gray hatched band is the $\pm 1 \sigma$ region around the SM predicted value.

\section{Conclusions}

The main conclusions from the overview presented here can be summarized as follows: the experimental studies on purely leptonic decays of the B mesons are, and will be, one of the most powerful tools to search for New Physics.

Combined analyses made at the B factories on the charged current mode $B \rightarrow \tau \nu$ show a 2.4 $\sigma$ deviation from the expected Standard Model value of $B F\left(B^{-} \rightarrow \tau^{-} \bar{v}\right)$.

Combined results of the experiments at Tevatron in the flavor changing neutral current sector will push the sensitivity on $B F\left(B_{s} \rightarrow \mu^{+} \mu^{-}\right)$close to SM predicted value; any evidence before the end of the Tevatron operations $\left(10 \mathrm{fb}^{-1}\right)$ will be evidence of New Physics. Tevatron results, combined with other measures, could severely constrain some New Physics models, such as mSUGRA, even before the end of the Tevatron Run-II.

In general, the accuracy on measurements on rare purely leptonic B decays is limited by statistics: sizable improvements with respect to present situation will be achieved at LHC and at the future Super B factories; the latter could increase the accuracy on $B F\left(B^{-} \rightarrow \tau^{-} \bar{v}\right)$ by a factor almost 10; the LHCb experiment could push the sensitivity on the $B F\left(B_{s} \rightarrow \mu^{+} \mu^{-}\right)$measurement below the Standard Model predicted value.

\section{References}

[1] G.Burdman and I.Shipsey, Ann.Rev.Nucl.Part.Sci.53:431-499,2003

[2] The CDF Collaboration, CDF public note 9226.

[3] I.Adachi et al. (Belle Collaboration), arXiv:0809.3834v1

[4] The BaBar Collaboration, arXiv:0809.4027v1

[5] B. Aubert et al. (BaBar Collaboration), Phys.Rev. D77, 011107 (2008)

[6] K.IKado et al. (Belle Collaboration), Phys.Rev.Lett. 97 251802, (2006)

[7] http: //ckmfitter.in2p3.fr/plots_Moriond09/ckmEval_results.html 
[8] S.-W. Hou, Phys.Rev. D48, 2342 (1993)

[9] S.Trine, arXiv:0810.3633v1

[10] A.J. Buras, arXiv:0904.4917v1

[11] T.Aaltonen et al. (CDF Collaboration), Phys.Rev.Lett. 100, 101802, (2008)

[12] V.M.Abazov et al. (D0 Collaboration), D76, 092001 (2007)

The D0 Collaboration, public note D0 5344-conf.

[13] B.Aubert et al. (BaBar Collaboration), Phys.Rev. D77, 032007 (2008)

[14] The D0 Collaboration, public note D0 5906-conf.

[15] R.Arnowitt et al., Phys.Lett. B538 (2002) 121

[16] Super B Conceptual Design Report, arXiV:0709.0451v2

[17] J.Albert et al., arXiV:physics/12235v1

[18] E.Conte, Nucl.Phys. B (Proc.Suppl.) 186 (2009) 411 